\title{
Hyperons in the relativistic mean-field approach to asymmetric nuclear matter
}

\author{
J. Kotulič Bunta* and Štefan Gmucd đ̈ \\ Institute of Physics, Slovak Academy of Sciences \\ Dubravska cesta 9, SK-842 28 Bratislava, Slovak Republic
}

(Dated: July 30, 2018)

\begin{abstract}
Relativistic mean-field theory with $\delta$ meson, nonlinear isoscalar self-interactions and isoscalarisovector cross interaction terms with parametrizations obtained to reproduce Dirac-BruecknerHartree-Fock calculations for nuclear matter is used to study asymmetric nuclear matter properties in $\beta$-equilibrium, including hyperon degrees of freedom and (hidden) strange mesons. Influence of cross interaction on composition of hyperon matter and electron chemical potential is examined. Softening of nuclear equation of state by the cross interactions results in lowering of hyperonization, although simultaneously enhancing a hyperon-induced decrease of the electron chemical potential, thus indicating further shift of a kaon condensate occurence to higher densities.
\end{abstract}

PACS numbers: 21.30.Fe, 21.65.+f, 24.10.Cn, 24.10.Jv

\section{INTRODUCTION}

Nuclear matter in $\beta$-equilibrium is one of the main objects of interest of nuclear physics with direct astrophysical implications.

Several theoretical models were developed for describing the nuclear matter. A direct connection between the fundamental nucleon-nucleon interaction and nuclear matter properties is provided by the Dirac-BruecknerHartree-Fock (DBHF) theory [1, 2, 3] which successfully describes properties of symmetric and also asymmetric nuclear matter. However, this sophisticated approach cannot be applied to finite nuclei calculations yet. Thus, other approaches are still relevant. Due to its elegant framework, the relativistic mean-field (RMF) theory [4, 5, 6] has been widely and successfully applied to wide range of finite nuclei. In order to eliminate phenomenological nature of RMF theory the free parameters can be fitted to the more fundamental DBHF approach 7], thus obtaining effective DBHF parametrization applicable also to finite nuclei. An alternative way is using the density dependent relativistic hadron theory [8] .

In recent years a significant progress in development of experimental techniques and devices was achieved. Modern accelerators enabled study of high energy ion collisions producing nuclei with high isospin asymmetry and nuclear densities higher than those occurring in normal nuclei. Therefore, a special attention was paid to a proper asymmetry description, e.g., an enhancement of isovector meson sector by $\delta$ meson introduction was performed [9]. However, better improvement of density dependence of symmetry energy was achieved by including of the scalar-vector cross interaction (VCI) term [10].

In addition to nuclear physics research also astrophysical phenomena are starting to provide relevant data with an accuracy needed to constrain the equation of state

\footnotetext{
*Electronic address: juraj.bunta@savba.sk
}

${ }^{\dagger}$ Electronic address: gmuca@savba.sk
(EOS) of nuclear matter. Neutron star masses and radii may be able to provide such constraints, due to their essential dependence on EOS, and many works deal with such calculations using DBHF approach 11, 12, 13], RMF theory 14, 15, or density dependent hadron field theory [16].

Since in the neutron star interior we expect densities of several times of the nuclear saturation density, extrapolation of models to high density region is inevitable. Classical view of matter as consisting of protons, neutrons and electrons is thus insufficient and more realistic composition is needed. At higher densities the $K^{-}$-condensate [17], quark deconfinement [18] and/or hyperons [19] are possible to appear.

After theoretical suggestion of the hyperon [20] and hypernuclei 21] appearance in high density nuclear matter many works paid attention to them, e.g., 22, 23, 24, 25], and experimental effort has started to obtain empirical data 26]. However, hyperon-meson coupling constants still remain uncertain. A partial exception is the $\Lambda$ hyperon where some constraint of its bindings is possible 27]. Even poorer knowledge we have about the $\Sigma^{-}$nucleon interaction. There is an absence of bound $\Sigma^{-}$ hypernuclear states 28] which might be a support for a high density repulsion of $\Sigma^{-}$-nucleon force as indicated by extrapolated atomic data 29]. However, this is still an open question. In any case, it seems to have only a weak effect on bulk properties of hyperon matter [30, 31. due to similar influence of the heavier $\Xi^{-}$hyperon, which (same as the $\Lambda$ hyperon) feels attractive potential 32]. Previous deducing of the $\Lambda \Lambda$ interaction from older double $\Lambda$ hypernuclei data suggested a highly attractive potential [33], while the very recent experimental observation of a ${ }_{\Lambda \Lambda}^{6} \mathrm{He}$ double hypernucleus demonstrated 34] that $\Lambda \Lambda$ interaction is only weakly attractive. With respect to all these facts, we used hyperon-nucleon and hyperon-hyperon coupling constants derived from $\mathrm{SU}(6)$ quark model for vector and isovector meson sector. Hyperon couplings to scalar mesons were fitted to reproduce experimental data for hypernuclei and double hypernuclei (see the next section for more details). 
Recently, the RMF parametrization of DBHF results for nuclear matter, including isovector scalar $\delta$ meson as well as scalar-vector cross interactions 35], was obtained. The results indicate a strong influence of cross interaction on the density dependence of symmetry energy. The goal of this paper is an application of this parametrizations also to asymmetric nuclear matter with hyperons, and thus to study the effect of additional degrees of freedom on a composition of dense matter. The theoretical framework described in the Section II. is followed by presentation and discussion of the obtained results in the Section III. Conclusions are summarized in the Section IV.

\section{THE MODEL}

We start with the Lagrangian density that introduces baryon field $\psi_{B}$, lepton fields $\psi_{e^{-}}$and $\psi_{\mu^{-}}$, isoscalarscalar meson field $\sigma$, isoscalar-vector meson field $\omega$, isovector-vector meson field $\boldsymbol{\rho}$, isovector-scalar meson field $\boldsymbol{\delta}$ (pion field does not contribute because of its pseudoscalar nature, and nuclear matter is parity invariant). Hidden strange scalar meson $\sigma^{*}$ and vector meson $\phi$ are introduced in order to better simulate hyperon-hyperon attraction observed in $\Lambda \Lambda$ hypernuclei [50]. Thus, the Lagrangian density takes a form,

$$
\begin{gathered}
\mathcal{L}\left(\psi_{B, e^{-}, \mu^{-}}, \sigma, \omega, \boldsymbol{\rho}, \boldsymbol{\delta}, \sigma^{*}, \phi\right) \\
=\sum_{B} \bar{\psi}_{B}\left[\boldsymbol{\gamma}_{\mu}\left(i \partial^{\mu}-g_{\omega} \omega^{\mu}-g_{\rho} \boldsymbol{\rho}_{\mu} \bar{\psi} \gamma^{\mu} \boldsymbol{\tau}-g_{\phi B} \phi^{\mu}\right)\right. \\
\left.-\left(M-g_{\sigma} \sigma-g_{\delta} \boldsymbol{\delta} \bar{\psi} \boldsymbol{\tau} \psi-g_{\sigma^{*} B} \sigma^{*}\right)\right] \psi_{B} \\
+\frac{1}{2}\left(\partial_{\mu} \sigma \partial^{\mu} \sigma-m_{\sigma}^{2} \sigma^{2}\right)-\frac{1}{3} b_{\sigma} M\left(g_{\sigma} \sigma\right)^{3}-\frac{1}{4} c_{\sigma}\left(g_{\sigma} \sigma\right)^{4} \\
-\frac{1}{4} \omega_{\mu \nu} \omega^{\mu \nu}+\frac{1}{2} m_{\omega}^{2} \omega_{\mu} \omega^{\mu}+\frac{1}{4} c_{\omega}\left(g_{\omega}^{2} \omega_{\mu} \omega^{\mu}\right)^{2} \\
+\frac{1}{2}\left(\partial_{\mu} \boldsymbol{\delta} \partial^{\mu} \boldsymbol{\delta}-m_{\delta}^{2} \boldsymbol{\delta}^{2}\right)+\frac{1}{2} m_{\rho}^{2} \boldsymbol{\rho}_{\mu} \cdot \boldsymbol{\rho}^{\mu}-\frac{1}{4} \boldsymbol{\rho}_{\mu \nu} \cdot \boldsymbol{\rho}^{\mu \nu} \\
+\frac{1}{2} \Lambda_{V}\left(g_{\rho}^{2} \boldsymbol{\rho}_{\boldsymbol{\mu}} \cdot \boldsymbol{\rho}^{\mu}\right)\left(g_{\omega}^{2} \omega_{\mu} \omega^{\mu}\right) \\
+\frac{1}{2}\left(\partial_{\mu} \sigma^{*} \partial^{\mu} \sigma^{*}-m_{\sigma^{*}}^{2} \sigma^{* 2}\right)+\frac{1}{2} m_{\phi}^{2} \phi_{\mu} \phi^{\mu}-\frac{1}{4} \phi_{\mu \nu} \phi^{\mu \nu} \\
+\sum_{e, \mu} \bar{\psi}_{e, \mu}\left(i \gamma_{\mu} \partial^{\mu}-m_{e, \mu}\right) \psi_{e, \mu}
\end{gathered}
$$

where antisymmetric field tensors are given by

$$
\begin{aligned}
\omega_{\mu \nu} & \equiv \partial_{\nu} \omega_{\mu}-\partial_{\mu} \omega_{\nu}, \\
\boldsymbol{\rho}_{\mu \nu} & \equiv \partial_{\nu} \boldsymbol{\rho}_{\mu}-\partial_{\mu} \boldsymbol{\rho}_{\nu}, \\
\phi_{\mu \nu} & \equiv \partial_{\nu} \phi_{\mu}-\partial_{\mu} \phi_{\nu},
\end{aligned}
$$

and the symbols used have the same meaning as in Ref. [35].

The nucleon-meson coupling constants as well as isoscalar self-interaction couplings and the vector cross interaction coupling were taken from our previous work 35]. Coupling constants of hyperons to the vector and isovector mesons as well as vector strange mesons are derived from the SU(6) simple quark model and are of the following form [37]:

$$
\begin{gathered}
\frac{1}{3} g_{\omega N}=\frac{1}{2} g_{\omega \Lambda}=\frac{1}{2} g_{\omega \Sigma}, \\
g_{\rho N}=\frac{1}{2} g_{\rho \Sigma}, \quad g_{\rho \Lambda}=0 \\
g_{\delta N}=\frac{1}{2} g_{\delta \Sigma}, \quad g_{\delta \Lambda}=0 \\
g_{\phi \Lambda}=g_{\phi \Sigma}=-\frac{\sqrt{2}}{3} g_{\omega N}, \quad g_{\phi N}=0 .
\end{gathered}
$$

Remaining couplings of hyperons to scalar mesons are adjusted to reproduce the hypernuclear potentials in saturated nuclear matter. A recent analysis [38] showed that $\Sigma^{-}$can feel repulsion in nuclear matter, thus leading to a strong suppression of its abundance in $\beta$-stable hyperon matter. However, for our purposes it is of a little importance because it has only a minor effect on bulk hyperon matter properties, as was shown in Ref. [30]. The matter is dominated by nucleons until high densities where universal short-range forces are expected to take precedence over the specific baryon identities 31]. Therefore, we have taken into account the following recent potential depths [27, 39]:

$$
U_{\Lambda}^{(N)}=-28 \mathrm{MeV}, U_{\Sigma}^{(N)}=+20 \mathrm{MeV}
$$

Finally, the hyperon couplings to scalar strange mesons are fixed by potential well of the $\Lambda$-hyperon in $\Lambda$ hyperonic matter deduced from the recent double- $\Lambda$ hypernuclear data [34], as estimated by Song [36].

$$
U_{\Lambda}^{(\Lambda)}=-5 \mathrm{MeV}
$$

Application of Euler-Lagrange equations to the Lagrangian (1) leads to equations of motion. Baryons fulfill the Dirac equation,

$$
\begin{gathered}
\sum_{B}\left[\gamma_{\mu}\left(i \partial^{\mu}-g_{\omega} \omega^{\mu}-g_{\rho} \boldsymbol{\rho}_{\mu} \cdot \boldsymbol{\tau}-g_{\phi B} \boldsymbol{\phi}^{\mu}\right)\right. \\
\left.-\left(M-g_{\sigma} \sigma-g_{\delta} \boldsymbol{\delta} . \boldsymbol{\tau}-g_{\sigma^{*} B} \sigma^{*}\right)\right] \psi_{B}=0
\end{gathered}
$$

while isoscalar $\sigma, \omega$, isovector $\delta, \rho$ and strange meson fields $\sigma^{*}$ and $\phi$ are described by Klein-Gordon and Proca equations, respectively, 


$$
\begin{aligned}
\left(\partial_{\mu} \partial^{\mu}+m_{\sigma}^{2}\right) \sigma= & g_{\sigma}\left[\sum_{B} \frac{g_{\sigma B}}{g_{\sigma}} \rho_{B}^{S}\right. \\
& -b_{\sigma} M\left(g_{\sigma} \sigma\right)^{2} \\
& \left.-c_{\sigma}\left(g_{\sigma} \sigma\right)^{3}\right], \\
\partial_{\mu} \omega^{\mu \nu}+m_{\omega}^{2} \omega^{\nu}= & g_{\omega}\left[\sum_{B} \frac{g_{\omega B}}{g_{\omega}} \rho_{B}^{B}\right. \\
& -c_{\omega} g_{\omega}^{3}\left(\omega_{\mu} \omega^{\mu} \omega^{\nu}\right) \\
& \left.-g_{\rho}^{2} \boldsymbol{\rho}_{\boldsymbol{\mu}} \cdot \boldsymbol{\rho}^{\mu} \Lambda_{V} g_{\omega} \omega_{\mu}\right], \\
\partial_{\mu} \boldsymbol{\rho}^{\mu \nu}+m_{\rho}^{2} \boldsymbol{\rho}^{\nu}= & g_{\rho}\left[\sum_{B} \frac{g_{\rho B}}{g_{\rho}} \rho_{B}^{B} \boldsymbol{\tau}\right. \\
& \left.-g_{\rho} \boldsymbol{\rho}_{\boldsymbol{\mu}} \Lambda_{V} g_{\omega}^{2} \boldsymbol{\omega}_{\mu} \boldsymbol{\omega}^{\mu}\right], \\
\left(\partial_{\mu} \partial^{\mu}+m_{\delta}^{2}\right) \boldsymbol{\delta}= & g_{\delta} \sum_{B} \frac{g_{\delta B}}{g_{\delta}} \rho_{B}^{S} \boldsymbol{\tau}, \\
\left(\partial_{\mu} \partial^{\mu}+m_{\sigma^{*}}^{2}\right) \sigma^{*}= & g_{\sigma^{*} \Lambda} \sum_{B} \frac{g_{\sigma^{*} B}}{g_{\sigma^{*} \Lambda}} \rho_{B}^{S}, \\
\partial_{\mu} \phi^{\mu \nu}+m_{\phi}^{2} \phi^{\nu}= & g_{\phi \Lambda} \sum_{B} \frac{g_{\phi B}}{g_{\phi \Lambda}} \rho_{B}^{B} .
\end{aligned}
$$

This set of nonlinear equations is solved in the meanfield approach, i.e., operators of meson fields are replaced by their expectation values. Additionally, a no-sea approximation is considered, which doesn't take account of the Dirac sea of negative energy states.

Static, homogenous, infinite nuclear matter allows us to consider some simplifications due to translational invariance and rotational symmetry of the nuclear matter. This causes the expectation values of spacelike components of vector fields vanish and only zero components, $\rho_{0}, \omega_{0}$, and $\phi_{0}$, remain. In addition, due to rotational invariance around the third ax of isospin space only the third component of isovector fields $\rho^{(3)}$ and $\delta^{(3)}$ survive. After reducing of the equation of motion meson field potentials follow directly:

$$
\begin{aligned}
U_{\sigma} \equiv & -g_{\sigma} \bar{\sigma}=-\frac{g_{\sigma}^{2}}{m_{\sigma}^{2}}\left[\sum_{B} \frac{g_{\sigma B}}{g_{\sigma}} \rho_{B}^{S}\right. \\
& \left.-b_{\sigma} M\left(g_{\sigma} \bar{\sigma}\right)^{2}-c_{\sigma}\left(g_{\sigma} \bar{\sigma}\right)^{3}\right], \\
U_{\omega} \equiv & g_{\omega} \bar{\omega}_{0}=\frac{g_{\omega}^{2}}{m_{\omega}^{2}}\left[\sum_{B} \frac{g_{\omega B}}{g_{\omega}} \rho_{B}^{B}\right. \\
& \left.-c_{\omega}\left(g_{\omega} \bar{\omega}_{0}\right)^{3}-U_{\rho}^{2} \Lambda_{V}\left(g_{\omega} \bar{\omega}_{0}\right)\right], \\
U_{\rho} \equiv & g_{\rho} \bar{\rho}_{0}^{(3)}=\frac{g_{\rho}^{2}}{m_{\rho}^{2}} \bar{\psi} \gamma^{0} \tau_{3} \psi= \\
& \frac{g_{\rho}^{2}}{m_{\rho}^{2}}\left[\sum_{B} \frac{g_{\rho B}}{g_{\rho}} \rho_{B}^{B} \tau_{3 B}-g_{\rho} \bar{\rho}_{0}^{(3)} \Lambda_{V} U_{\omega}^{2}\right],
\end{aligned}
$$

$$
\begin{aligned}
U_{\delta} & \equiv-g_{\delta} \bar{\delta}^{(3)}=-\frac{g_{\delta}^{2}}{m_{\delta}^{2}} \sum_{B} \frac{g_{\delta B}}{g_{\delta}}\left(\rho_{B}^{S} \tau_{3 B}\right) \\
U_{\sigma^{*}} & \equiv-g_{\sigma^{*} \Lambda} \bar{\sigma}^{*}=-\frac{g_{\sigma^{*} \Lambda}^{2}}{m_{\sigma^{*}}^{2}} \sum_{B} \frac{g_{\sigma^{*} B}}{g_{\sigma^{*} \Lambda}} \rho_{B}^{S} \\
U_{\phi} & \equiv g_{\phi \Lambda} \bar{\phi}_{0}=\frac{g_{\phi \Lambda}^{2}}{m_{\phi}^{2}} \sum_{B} \frac{g_{\phi B}}{g_{\phi \Lambda}} \rho_{B}^{B}
\end{aligned}
$$

where $\tau_{3 p}=1, \tau_{3 n, \Sigma^{-}}=-1, \tau_{3 \Lambda^{0}}=0$ are isospin projections for baryons. Scalar density $\rho_{S}$ is expressed as the sum of baryon $\left(B=p, n, \Lambda^{0}, \Sigma^{-}\right)$contributions

$$
\rho_{B}^{S}=\frac{2 J_{B}+1}{(2 \pi)^{3}} \int_{0}^{k_{B}} d^{3} k \frac{M_{B}^{*}}{\left(\boldsymbol{k}^{2}+M_{B}^{* 2}\right)^{1 / 2}} .
$$

In Eq. (18) $k_{B}$ is baryons' Fermi momentum, $J_{B}$ corresponds to baryon spin and $M_{B}^{*}$ denotes baryon effective masses which can be written as

$$
M_{B}^{*}=M-g_{\sigma B} \bar{\sigma}-g_{\delta B} \bar{\delta}^{3} \tau_{3 B}-g_{\sigma^{*} B} \bar{\sigma}^{*} .
$$

Condensed scalar $\sigma, \delta$, and $\sigma^{*}$ meson fields generate a shift of baryon masses, in consequence of which nuclear matter is described as a system of pseudonucleons with masses $M_{B}^{*}$ moving in classical fields. Note that while $\sigma$ meson shifts all the baryon masses (even with different strength for nucleons and hyperons), $\delta$ meson field is responsible for splitting of effective masses of baryons with nonzero isospin only, which is an important feature of $\delta$ meson influence on the nuclear matter saturation mechanism and its properties. Additionally, due to no interaction of strange mesons with nucleons, $\sigma^{*}$ meson shifts only hyperon masses.

Total baryon density is given by,

$$
\rho_{B}=\sum_{B} \rho_{B}^{B}=\sum_{B} \frac{2 J_{B}+1}{(2 \pi)^{3}} \int_{0}^{k_{B}} d^{3} k=\sum_{B} \frac{k_{B}^{3}}{3 \pi^{2}} .
$$

The momentum-energy tensor

$$
T_{\mu \nu}=-g_{\mu \nu} \mathcal{L}+\frac{\partial \Phi_{i}}{\partial \boldsymbol{x}^{\nu}} \frac{\partial \mathcal{L}}{\partial\left(\partial \Phi_{i} / \partial \boldsymbol{x}_{\mu}\right)},
$$

where $\Phi_{i}$ generally denotes physical fields, gives the energy density of the system as its zero component $\varepsilon=$ $\left\langle T_{00}\right\rangle$, and finally, the binding energy per nucleon is related to the energy density by,

$$
E_{b}=\frac{\varepsilon}{\rho_{B}}-M .
$$

While we are considering $\beta$-stable nuclear matter consisting of baryons, hyperons, and leptons, there have to be fulfilled equilibrium conditions of chemical potentials 
defined as Fermi energy of particles at the top of Fermi sea, for baryons taking a form,

$$
\mu_{B}=g_{\omega B} \bar{\omega}_{0}+g_{\rho B}{\overline{\rho_{0}}}^{(3)} \tau_{3 B}+g_{\phi B} \bar{\phi}_{0}+\sqrt{k_{B}^{2}+M_{B}^{*}{ }^{2}},
$$

and for leptons,

$$
\mu_{e, \mu}=\sqrt{k_{e, \mu}^{2}+m_{e, \mu}^{2}}
$$

Generally, the chemical potential equilibrium conditions can be written as,

$$
\mu_{B}=q_{b, B} \mu_{n}-q_{e, B} \mu_{e},
$$

where $q_{b, B}$ is baryon number of particles, and $q_{e, B}$ denotes electric charge of particles, giving a set of equilibrium equations,

$$
\begin{aligned}
\mu_{\Lambda} & =\mu_{n}, \\
\mu_{\Sigma^{-}} & =\mu_{n}+\mu_{e}, \\
\mu_{p} & =\mu_{n}-\mu_{e}, \\
\mu_{\mu} & =\mu_{e} .
\end{aligned}
$$

Simultaneously, charge neutrality:

$$
\rho_{p}=\rho_{e}+\rho_{\mu}+\rho_{\Sigma^{-}}
$$

and the total baryon density (20) have to be met.

\section{RESULTS AND DISCUSSION}

The reference 35 deals with the role of $\delta$ meson and vector cross interaction in asymmetric nuclear matter. The present work extends this study to hyperon matter in $\beta$-equilibrium.

Several different mean-field parametrizations are used, taken from the Ref. 35]. The first pair MA and MB (listed in Tab. I) results from the fit of DBHF nuclear matter calculations performed by Machleidt and coworkers 40]. Binding energy per nucleon for symmetric matter and neutron matter, and simultaneously symmetric matter isoscalar potentials (all results for Bonn A NN potential) were taken into account. They differ in degrees of freedom used - the second one does not include the vector cross interaction term. Couplings of hyperons to scalar meson were fitted to reproduce hypernuclei potential well given by Eq. (3), and strengths of strange scalar meson couplings are adjusted to fulfil potential depth from Eq. (4). Similarly the second parametrization pair LA and LB (also listed in Tab. I) reproduces DBHF results of Lee and co-workers 41] (also Bonn A potential), where, in addition to the first fit, also binding energies for several asymmetries were fitted together with symmetric matter isoscalar potentials. The next parametrizations HA - HD (which differ in inclusion or exclusion of cross interaction and $\delta$ meson) ensue from the fit of DBHF results of Huber and co-workers 42] (Bonn B potential), being fitted to binding energy per nucleon for several asymmetries, symmetric matter isoscalar potentials, and, unlike the previous two sets, also to proton and neutron isoscalar potentials, which enables an evaluation of the $\delta$ meson field contribution. Isoscalar $\sigma, \omega$ mesons with their self-interactions, isovector $\rho$ meson, $\rho-\omega$ cross interaction, and for relevant parametrization sets also $\delta$ meson, were used as degrees of freedom.

TABLE I: Parameter sets resulting from the fit 35] of Machleidt and co-workers [40] and Lee and co-workers 41] DBHF results (for nucleon-meson coupling constants). Hyperonscalar meson couplings resulting from the fit to hypernuclear potentials (3), (4) are also listed, hyperon-vector (isovector) meson couplings ensue directly from Eqs. (2).

\begin{tabular}{ccccc}
\hline \hline & MA & MB & LA & LB \\
\hline$g_{\sigma}^{2}$ & 106.85 & 112.27 & 103.91 & 102.11 \\
$g_{\omega}^{2}$ & 180.61 & 204.36 & 147.84 & 146.73 \\
$g_{\rho}^{2}$ & 18.445 & 9.493 & 17.432 & 9.670 \\
$b_{\sigma}$ & -0.00258 & -0.00298 & 0.000972 & 0.000836 \\
$c_{\sigma}$ & 0.0115 & 0.0133 & 0.00127 & 0.00124 \\
$c_{\omega}$ & 0.0158 & 0.0204 & 0.00542 & 0.00519 \\
$\Lambda_{V}$ & 0.2586 & & 0.1879 & \\
$\chi^{2} / N$ & 2.76 & 9.95 & 1.69 & 2.62 \\
\hline$g_{\sigma \Lambda} / g_{\sigma N}$ & 0.602 & 0.599 & 0.602 & 0.601 \\
$g_{\sigma \Sigma} / g_{\sigma N}$ & 0.472 & 0.473 & 0.475 & 0.476 \\
$g_{\sigma * \Lambda(\Sigma)} / g_{\sigma N}$ & 0.646 & 0.662 & 0.603 & 0.605 \\
\hline \hline
\end{tabular}

TABLE II: Parameter sets resulting from the fit 35] to Huber and co-workers DBHF results [42]. Hyperon couplings are obtained analogically as in Tab. []

\begin{tabular}{ccccc}
\hline \hline & $\mathrm{HA}$ & $\mathrm{HB}$ & $\mathrm{HC}$ & $\mathrm{HD}$ \\
\hline$g_{\sigma}^{2}$ & 90.532 & 86.432 & 91.110 & 87.591 \\
$g_{\omega}^{2}$ & 108.95 & 106.89 & 109.26 & 107.61 \\
$g_{\rho}^{2}$ & 36.681 & 28.795 & 20.804 & 15.335 \\
$g_{\delta^{2}}$ & 28.739 & 25.170 & & \\
$b_{\sigma}$ & 0.00439 & 0.00338 & 0.00444 & 0.00357 \\
$c_{\sigma}$ & -0.00520 & -0.00378 & -0.00521 & -0.00398 \\
$c_{\omega}$ & -0.000142 & -0.00105 & -0.0000385 & -0.000775 \\
$\Lambda_{V}$ & 0.1065 & & 0.3481 & \\
$\chi^{2} / N$ & 2.05 & 3.80 & 5.85 & 6.89 \\
\hline$g_{\sigma \Lambda} / g_{\sigma N}$ & 0.605 & 0.600 & 0.606 & 0.601 \\
$g_{\sigma \Sigma} / g_{\sigma N}$ & 0.446 & 0.449 & 0.446 & 0.448 \\
$\left.g_{\sigma * \Lambda} \Lambda\right) / g_{\sigma N}$ & 0.576 & 0.582 & 0.576 & 0.580 \\
\hline \hline
\end{tabular}

By simultaneous calculation of the equilibrium conditions and meson field potentials the dependence of particle fractions on baryon density was obtained. We considered $\beta$-stable nuclear matter consisting of protons, neutrons, $\Sigma^{-}, \Lambda^{0}$ hyperons, electrons and muons. We did not take into account other types of hyperons because 


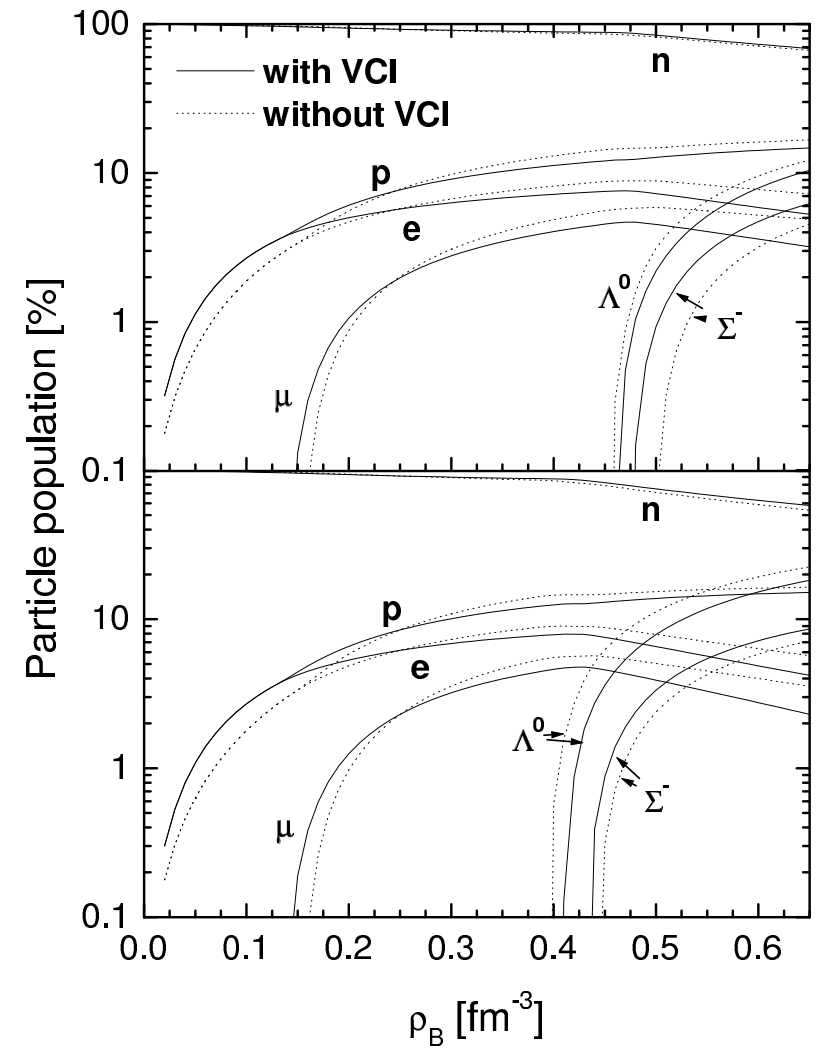

FIG. 1: Populations of particles in $\beta$-stable hyperon matter, calculated with parametrization sets which reproduce Dirac-Brueckner-Hartree-Fock results obtained by Machleidt et al. 40] [upper panel], and that of Lee et al. 41] [lower panel]. Solid lines correspond to the fit and equilibrium calculations performed with vector cross interactions (VCI) (MA, LA), dotted lines denotes calculations where cross interaction was excluded (MB, LB). Nucleon-meson parametrizations are taken from Ref. [35] and listed in Tab. [1]

the heaviest $\Delta^{\frac{ \pm}{0},++}$ seem to be suppressed [22, 43] and also other baryons $\left(\Sigma^{0}, \Xi^{0}\right)$ due to their electric charge or higher rest mass may appear only at very high densities, or may not appear at all 44]. Since the parametrizations from Ref. [35] result from fits up to densities around $0.33 \mathrm{fm}^{-3}$, the reliability of the extrapolation to such a high density region decreases. Moreover, it seems that at least some important matter properties are more affected by total hyperonization than by hyperon subtypes themselves, which is indicated for example by very similar influence of hyperons on electron chemical potential with presence or with total exclusion of $\Sigma^{-}$(due to its possible strong repulsion in nuclear medium), which can be compensated by $\Xi^{-}[30]$. Therefore, the particular abundance of baryon species is not very important 31.

In Fig. 1 the results for parametrizations MA (MB) (upper panel) and LA (LB) (lower panel) are shown. Starting from almost pure neutron matter with rising total baryon density the nuclear matter is enriched by protons and, consequently, due to charge neutrality also by electrons. Subsequently, the electron chemical poten- tial is rising and when it reaches value of the rest mass of muon it become possible to turn electron into muon. This happens already closely below the saturation density at $\rho_{B}=0.14 \mathrm{fm}^{-3}$ and causes also slight growth of proton fraction rise. With further rise of density it turns up energetically favorable to turn nucleons with high kinetic energy into the heavier baryon resonances. The first hyperon appearing is the $\Lambda^{0}$ at density $0.46 \mathrm{fm}^{-3}$.

In many older works (e.g., 16, 30, 31, 44, 45]) it is $\Sigma^{-}$ hyperon that appear as the first one. This was due to its negative electric charge and zero isospin of the lightest $\Lambda^{0}$ - the electron chemical potential together with $\rho$ meson field affecting $\Sigma^{-}$was higher than $\Sigma^{-}$and $\Lambda^{0}$ effective mass difference. However, this was in the case of assuming the negative potential well which feels $\Sigma^{-}$in nuclear matter, whereas later investigation shows that it feels more probably a strong repulsion. Thus, the $\Lambda^{0}$ onset is followed by $\Sigma^{-}$hyperon at density $0.48 \mathrm{fm}^{-3}$. Nevertheless, we have to note that quantitative value of the threshold densities depends strongly on poorly known nucleonhyperon coupling constants and their better knowledge will improve correspondence of theory with reality. In any case, the onset of hyperons has distinct influence on nucleon and lepton populations. Hyperons due to the chemical potential relations heighten proton fraction following by a fall of number of both leptons in order to hold the charge neutrality of matter. The lepton population reduction is a consequence of the local non-conservation of lepton number (neutrinos escape from matter). These general characteristics of density dependence of $\beta$-stable hyperon matter are (except the $\Sigma^{-}$repulsion) qualitatively in accordance with the above mentioned works.

For examination of the vector cross interactions the results for parametrization without them (MB) are drawn in the same plot by dotted lines. Since VCI are softening the equation of state of nuclear matter, it generally enforces neutron population to the prejudice of protons with slight shift of negatively charged muons and $\Sigma^{-}$onset to the lower densities and that of neutral $\Lambda^{0}$ to the higher densities, thus reducing the onset density difference. Without the VCI muons appear at $0.16 \mathrm{fm}^{-3}, \Lambda^{0}$ at $0.45 \mathrm{fm}^{-3}$ and $\Sigma^{-}$at $0.50 \mathrm{fm}^{-3}$.

The lower panel of Fig. 11 shows populations for the second parametrization pair LA (LB) where the muons appear at $0.14 \mathrm{fm}^{-3}\left(0.16 \mathrm{fm}^{-3}\right), \Lambda^{0}$ at $0.41 \mathrm{fm}^{-3}(0.40$ $\left.\mathrm{fm}^{-3}\right)$, and $\Sigma^{-}$at $0.43 \mathrm{fm}^{-3}\left(0.44 \mathrm{fm}^{-3}\right)$, values in the brackets being for non-VCI case. The main difference in comparison with the previous parameter set is lowering the hyperon thresholds and generally richer hyperonization of matter. While in MA (MB) set the total hyperonization of matter at density $\rho_{B}=0.6 \mathrm{fm}^{-3}$ is $12.7 \%$ $(12.7 \%)$, in LA (LB) results it is $22.4 \%$ (25.1\%). This is a natural consequence of harder EOS in the second parametrization set.

The next parametrizations HA-D give outcome plotted in Fig. 2 Solid line in both panels denotes particle population density dependence with inclusion of both $\delta$ meson and VCI (HA). The influence of the VCI absence 


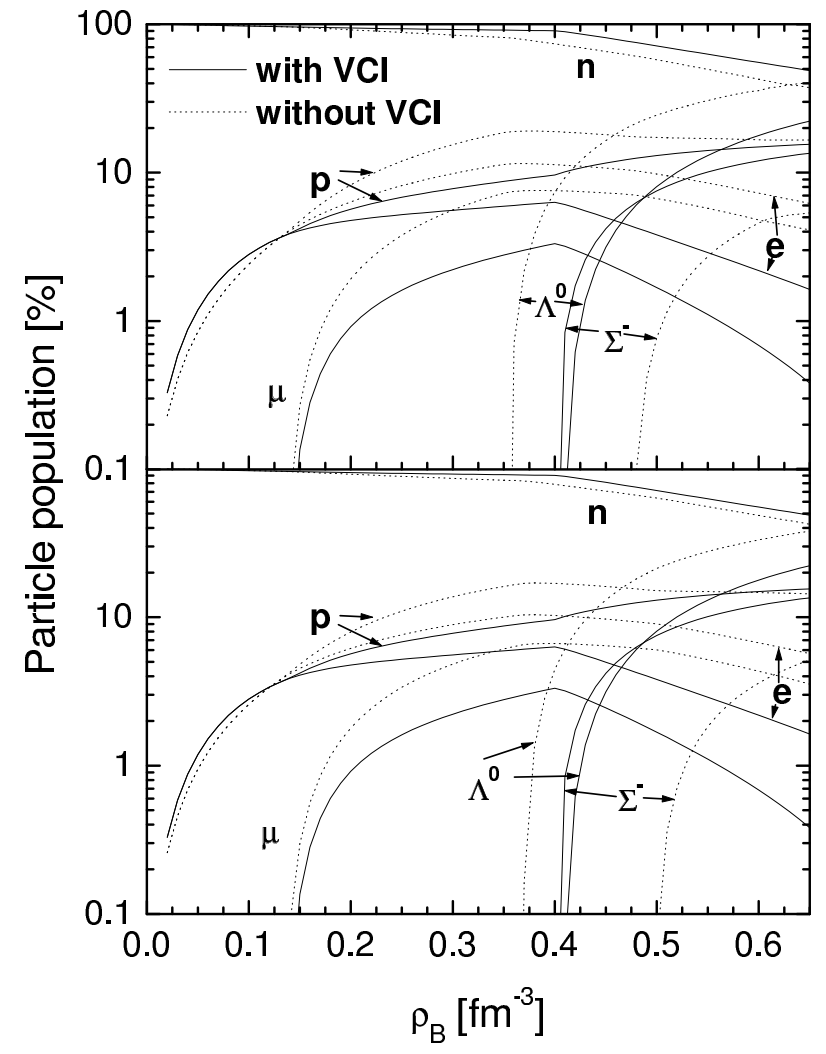

FIG. 2: Particle populations calculated similarly as in Fig. 11 with nucleon-meson parametrization sets taken from Ref. 35], here relisted in Tab. [I] which reproduces DBHFcalculated asymmetric nuclear matter properties performed by Huber et al. [42]. In both panels solid lines represent results of fit equilibrium calculations performed with $\delta$ meson as well as vector cross interaction (HA). Dotted lines in the upper panel are results with $\delta$ meson inclusion but without VCI (HB), whereas dotted lines in the lower panel represents populations with parametrization which does account of neither $\delta$ meson nor VCI (HD).

with simultaneous $\delta$ meson inclusion is seen from dotted lines in the upper panel (HB) while an analogical situation in $\delta$ meson absence can be seen from dotted lines in the lower panel (HD). The VCI effect is more distinct than in the previous two parameter sets. First, in density range roughly from one to two times saturation density there is a strong shortage of protons when VCI is present, independently on the $\delta$ meson. Second important point is a shift of $\Lambda^{0}$ threshold to higher densities from 0.36 $\mathrm{fm}^{-3}$ to $0.41 \mathrm{fm}^{-3}$ with $\delta$ meson or from $0.37 \mathrm{fm}^{-3}$ to $0.41 \mathrm{fm}^{-3}$ without it as well as a shift of $\Sigma^{-}$threshold to lower densities. The influence of VCI on hyperon thresholds is in this case so strong that in spite of the strong repulsion of $\Sigma^{-}$hyperon in nuclear matter it appears as the first hyperonic species. Consequently, the third effect of VCI is more rapid deleptonization of matter - at density $0.6 \mathrm{fm}^{-3}$ there are $0.69 \%(4.85 \%)$ of muons and $2.19 \%(7.28 \%)$ of electrons in HA (HB) case, while $2.75 \%$ $(4.00 \%)$ of muons and $4.87 \%(6.34 \%)$ of electrons in LA

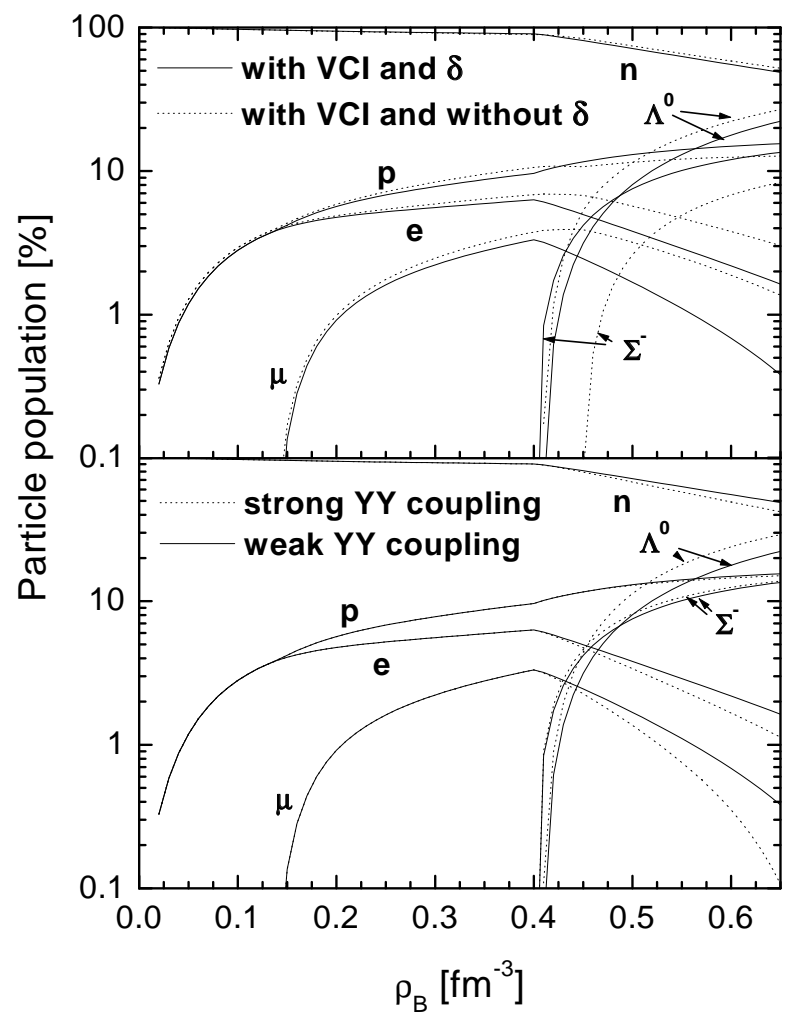

FIG. 3: Influence of isovector $\delta$ meson [upper panel] and strange mesons [lower panel] on the particle populations. Solid lines represent calculations with both $\delta$ and strange mesons (HA) with weak YY interactions, dotted lines in the upper panel are populations without $\delta$ meson (HC), and dotted lines in the lower panel denote the results where YY interactions were strengthened $\left(U_{\Lambda}^{(\Lambda)}=-20 \mathrm{MeV}\right)$.

(LB) parametrizations, and $3.60 \%(5.22 \%)$ of muons and $5.88 \%(7.75 \%)$ of electrons in MA (MB) parameter sets. It is clear that the VCI play primary role in equilibrated matter and it is an indispensable physical degree of freedom; this supports conclusions from [35].

To evaluate independently an effect of the isoscalar scalar degree of freedom the particle populations are drawn again in Fig. 3. upper panel, both VCI including and explicitly with (solid lines, HA) and without (dotted ones, $\mathrm{HC}) \delta$ meson. It influences mostly the onset of $\Sigma^{-}$ hyperon (isovector $\delta$ meson does not interact with $\Lambda^{0}$ ), however, not to such amount as VCI. Moreover, the total hyperonization is also affected only slightly - presence of $\delta$ meson increases it from $29.0 \%$ only to $29.8 \%$. One can conclude that in spite of its isospin nature the role of $\delta$ meson is of a secondary importance also in highly asymmetric matter. This is in agreement with conclusions of Ref. [45].

In the same figure (lower panel) the role of the strength of hyperon-hyperon interaction mediated by strange $\sigma^{*}$ and $\phi$ mesons is examined. Solid lines represent results employing weak YY interaction (Eq. (44) while dotted lines correspond to enforced YY coupling with potential 


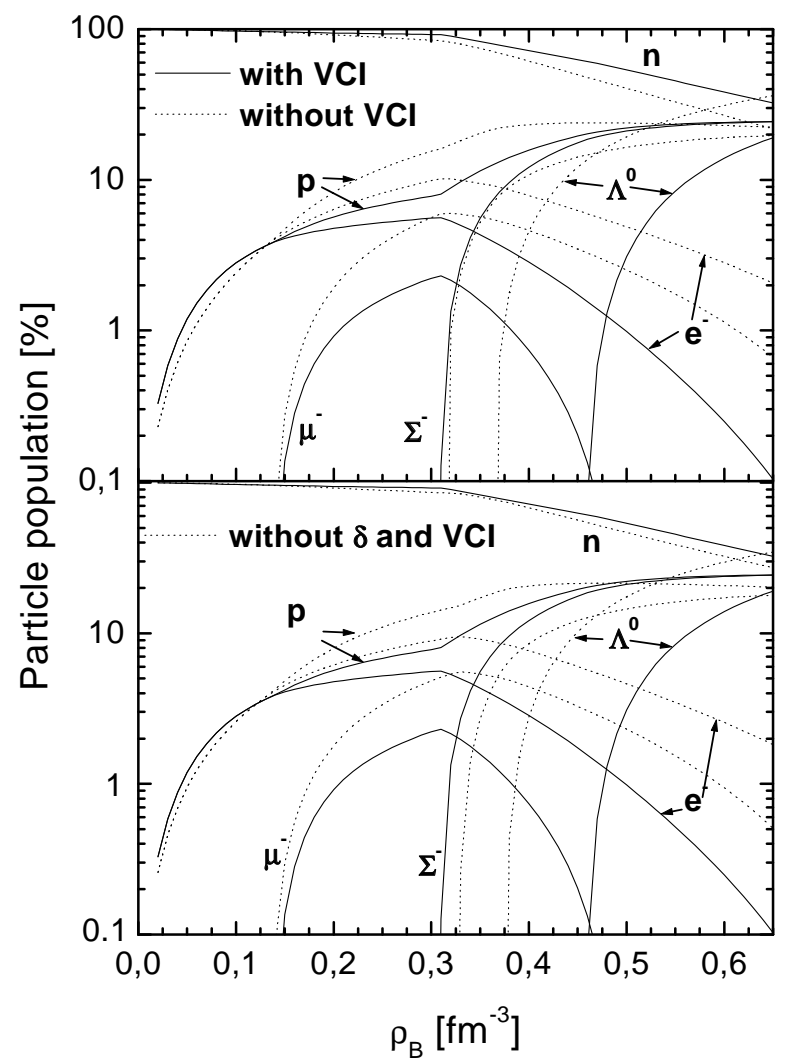

FIG. 4: Particle populations calculated using attractive potential for $\Sigma^{-}$hyperon in nuclear matter: $U_{\Sigma^{-}}^{(N)}=-30 \mathrm{MeV}$. Analogically to Fig. 2 in both panels solid lines represent results of fit equilibrium calculations performed with $\delta$ meson as well as vector cross interaction (HA). Dotted lines in the upper panel are results with $\delta$ meson inclusion but without VCI (HB), whereas dotted lines in the lower panel represent populations with parametrization which does account of neither $\delta$ meson nor VCI (HD).

well of $\Lambda$ hyperon in hyperonic matter $\mathrm{U}_{\Lambda}^{(\Lambda)}=-20 \mathrm{MeV}$. As strange mesons are not coupled to nucleons, their effect starts up above the $\Sigma^{-}$threshold density (which is not affected) and is only of a small magnitude. Another effect of strange mesons is a stabilization of matter at very high densities. For LA (B) negative nucleon effective masses appear above $1.89 \mathrm{fm}^{-3}\left(1.76 \mathrm{fm}^{-3}\right)$ which are changed to a positive value if $\sigma^{*}, \phi$ are introduced. Even there does not occur also negative electron chemical potential, this is in accordance with results of Ref. [45].

Results of calculations in the case of $\Sigma^{-}$hyperon feeling an attractive interaction (negative potential well $\mathrm{U}_{\Sigma^{-}}^{(N)}=-30 \mathrm{MeV}$ ) which seemed to be realistic in older works, are drawn in Fig. [4 Analogically to Fig. 2 on both panels there are shown results for HA parametrization (with hyperon couplings modified to reproduce negative potential well of $\Sigma^{-}$) with solid lines. In the upper panel the dotted lines represent results from the parametrization HB (without VCI), and the dotted lines

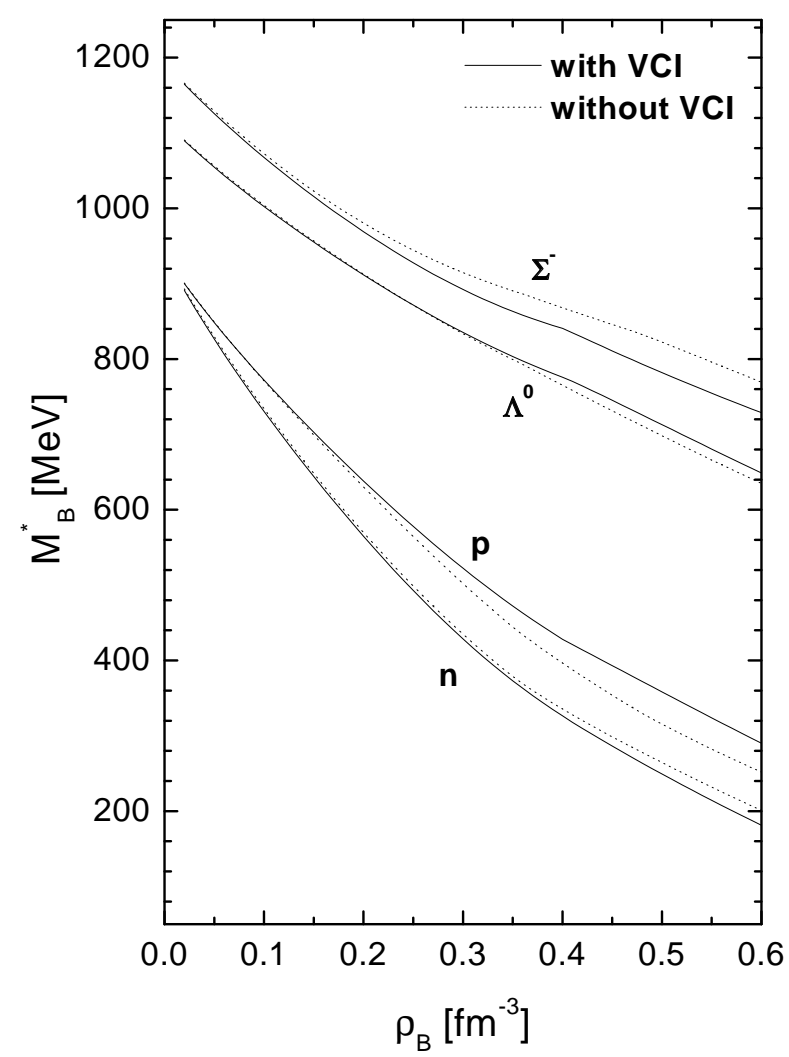

FIG. 5: Density dependence of the effective baryon masses in $\beta$-stable hyperon matter calculated with parametrization including $\delta$ meson, vector cross interaction and strange mesons, listed in Tab. II (solid lines, HA), and without VCI (dotted lines, HB).

in the lower panel correspond to parametrization HD (without VCI and $\delta$ meson). We can see that in spite of strong (and natural) decrease of threshold baryon density for $\Sigma^{-}$hyperon onset, the general features of VCI influence are the same. Also in this case VCI enhance the deleptonization process, lowers total hyperonization of matter and strongly affect population of protons in the middle-density region.

Besides not very strong effect of $\delta$ meson on the composition of $\beta$-stable matter, due to its isospin vector nature it has an impact on effective masses of baryons with nonzero isospin. It can be seen from Fig. 5 where nucleon masses are splitted. Nonetheless, in the VCI presence the $\delta$ potential saturation at about $55 \mathrm{MeV}$ occurs after hyperon appearance, as it flows from Fig. [6] This saturation is given by a balance of the total baryon density and by fractions of non-zero isospin baryons and additionally supports our conclusion and conclusion from Ref. 45 of small $\delta$ meson influence. Lower $\delta$ meson field in VCI absence (saturation at $30 \mathrm{MeV}$ ) is given partially by lower coupling of $\delta$ meson to nucleons $\left(g_{\delta}^{2} / g_{\delta \mathrm{VCI}}^{2}=0.88\right)$ needed for proper reproduction of DBHF calculations as well as by richer proton and $\Lambda^{0}$ and poorer neutron and $\Sigma^{-}$populations, thus resulting in more isospin symmet- 


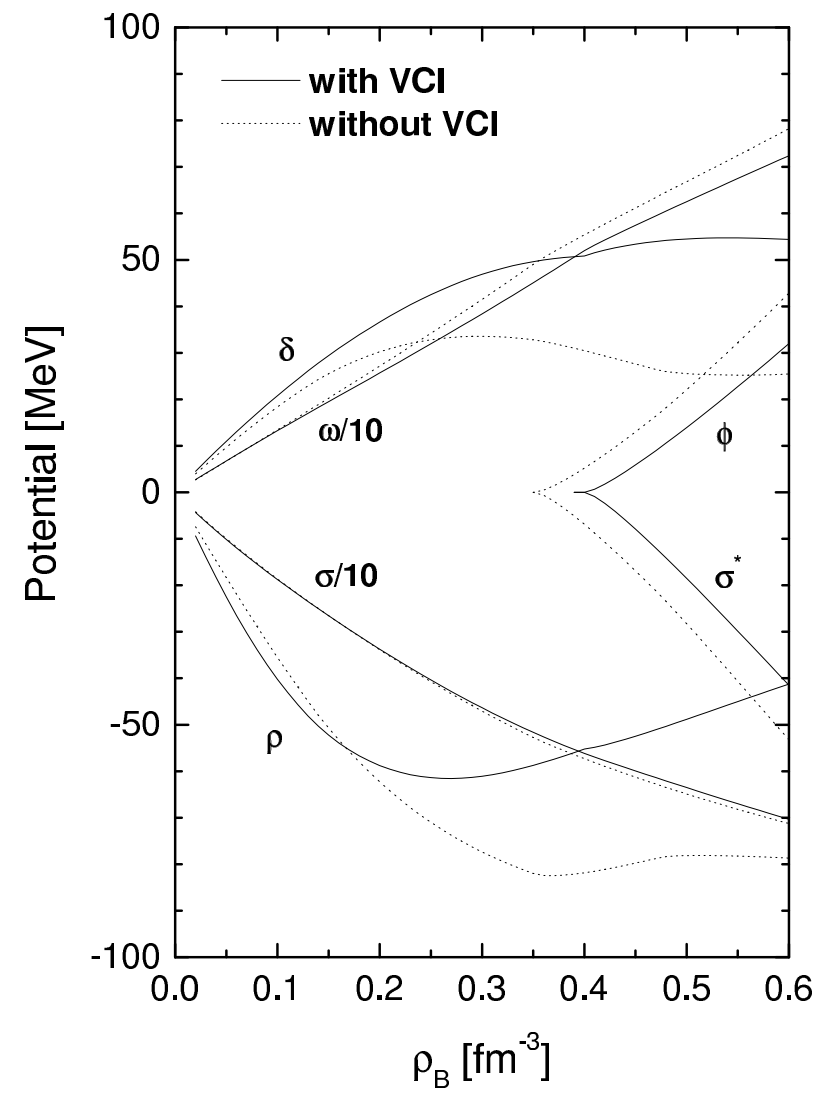

FIG. 6: Meson field potentials calculated with the same full parametrization set as in the previous figure (solid line, HA), and with exclusion of vector cross interaction (dotted line, HB). For practical purposes isoscalar $\sigma$ and $\omega$ fields are shown divided by factor 10 .

ric matter. Even stronger saturation mechanism occurs for the $\rho$ meson field, in this case it is a direct impact of cross interactions with $\omega$ field which also exhibits decrease, though due to much weaker $\rho$ field not leading to a saturation. Finally, since the VCI soften EOS, in their absence there is a higher hyperonization of matter - including (excluding) the isovector-scalar field it is 40.7 $\%(36.8 \%)$, compared to $29.8 \%(29.0 \%)$ at $\rho_{B}=0.6$ $\mathrm{fm}^{-3}$ if they are switched on, when weaker strange fields are also another natural consequence.

Another important feature of the hyperon onset is a strong change in the density dependence of electron chemical potential. Distinct decline of the electron Fermi momentum and concentration in matter after hyperonization is immediately followed by a drop of chemical potential which has serious consequences for an occurance of kaon condensation, as was first pointed out in Ref. 22]. Density dependence of the chemical potential of electrons in equilibrated matter for parametrizations examined in this paper are plotted in Fig. 7 The upper panel shows chemical potential resulting same as in the previous figures from parametrization with $\sigma, \omega, \rho$, and $\delta$ mesons with (HA) and without VCI (HB). The recent effective kaon mass resulting for nuclear matter [46] and

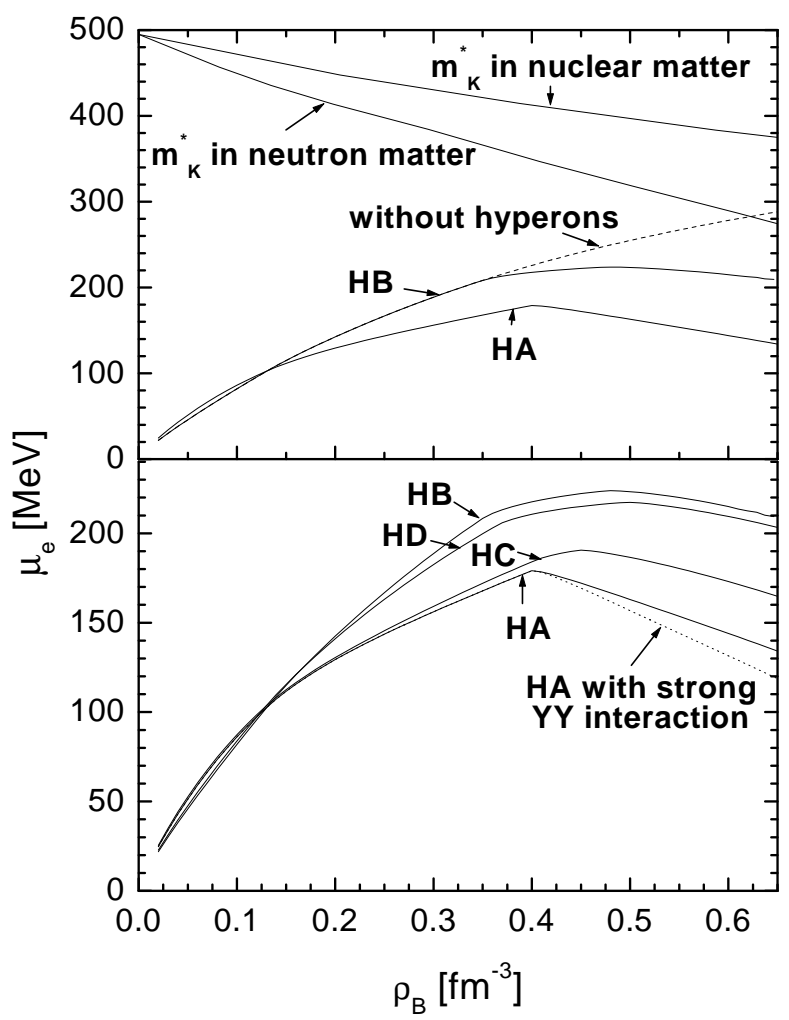

FIG. 7: Chemical potentials of electrons in $\beta$-stable hyperon matter obtained with parametrization with $\sigma, \omega, \rho, \delta$ mesons, isoscalar self-interactions, and vector cross interaction (Tab. II HA) and without cross interactions (HB), as well as density dependence of the electron chemical potential in matter without hyperons (dotted line). For comparison, the effective mass of $K^{-}$in nuclear matter (taken from [46]) and neutron matter [47] is shown. The lower panel shows the results for remaining parametrizations $\mathrm{HC}$ and $\mathrm{HD}$ and, additionally, the results of HA but without strange mesons.

neutron matter [47] is also plotted. However, its value is still an actual topic of research and at least for neutron matter may be even lower (around $200 \mathrm{MeV}$ at three times of saturation density), as suggest recent analysis [48] of experimental data 49]. If the electron chemical potential reaches effective kaon mass the kaon condensation occurs. Nevertheless, as could be expected, the electron chemical potential stops to rise after hyperonization (otherwise it would continually increase as shows dashed line) and starts to decline with increasing density.

The vector cross interaction affects both the maximum value of chemical potential and the slope of its decrease. The VCI also causes an immediate reduction of the chemical potential, while in their absence there is a mild rise after hyperon onset as can be better seen from the lower panel. There is more detailed comparison of different parameter sets. Without VCI, the electron chemical potential saturates at value about $224 \mathrm{MeV}$ and $\delta$ meson has only a weak effect (about $6 \mathrm{MeV}$ ), otherwise the saturation value is around $191 \mathrm{MeV}$ and even $179 \mathrm{MeV}$ with $\delta$ 
meson. Due to almost zero strange meson fields at breakpoint the stronger YY coupling has no influence on the saturation value and for higher densities it mildly fortifies VCI influence on the electron chemical potential. Additionally, we have performed analysis of the situation if $\Sigma^{-}$hyperon feels attractive potential in hyperon matter (or negative potential well) and it is important to note that in this case the effect of VCI is even stronger - it brings additional decrease of electron chemical potential approximately by $30 \%$ (for clarity these results are not shown in the Fig. (7). If $m_{K^{-}}=200 \mathrm{MeV}$ at $\rho=3 \rho_{0}$ verges on reality, it might not be clear if inclusion of hyperons themselves would prevent kaon condensation. Nevertheless, considering of VCI could additionally shift it to much higher densities, possibly already not relevant for neutron stars. Thus, VCI strongly supports hyperons in their role of kaon condensation reduction factor, although intensity of this effect depends on the exact kaon effective mass in nuclear medium as well as accurate hyperon-nucleon constants.

\section{SUMMARY}

In this work, several parametrizations of the relativistic mean-field theory reproducing three different DiracBrueckner-Hartree-Fock calculations for asymmetric nuclear matter are used to calculate properties of hyperon matter in $\beta$-equilibrium. Besides the isoscalar meson with their self-interactions and isovector $\rho$ meson, also isovector-scalar $\delta$ meson, and scalar-vector cross interactions are considered and their influence on composition of equilibrated hyperon matter and the electron chemical potential is studied. Supporting previous conclusions the results obtained indicate that VCI is an important degree of freedom with the distinct impact on both the composition of matter and the electron chemical potential. Since they soften nuclear equation of state, especially if the $\delta$ meson is present, they reduce hyperonization of matter and heighten population of neutrons, thus making the neutron star matter more neutron-rich. Notwithstanding, they simultaneously strongly support hyperons in their role of kaon condensation reduction factor, resulting in a lower saturation value of the electron chemical potential and also its more steep decreasing after the hyperon onset. That shifts the kaon condensation appearance to even higher densities and makes the neutron star matter more neutron-rich also from this point of view. It is interesting that neither stronger YY interaction nor attractive potential of $\Sigma^{-}$hyperon lessen this influence.

The next step is to apply this parametrizations also to neutron star properties calculation. Such a work is in progress.

\section{Acknowledgments}

This work was supported in part by the Slovak Grant Agency for Science VEGA under Grant No. 2/4098/04. Calculations were performed at the Computational Centre of Slovak Academy of Sciences.

\section{APPENDIX}

[1] R. Brockmann and R. Machleidt, Phys. Lett. B 149, 283 (1984).

[2] B. ter Haar and R. Malfliet, Phys. Rep. 149, 207 (1987).

[3] F. de Jong and H. Lenske, Phys. Rev. C 57, 3099 (1998).

[4] B. D. Serot and J. D. Walecka, Adv. Nucl. Phys. 16, 1 (1986).

[5] B. D. Serot and J. D. Walecka, Int. J. Mod. Phys. E 6, 515 (1997).

[6] A. R. Bodmer, Nucl. Phys. A292, 703 (1991).

[7] S. Gmuca, Nucl. Phys. A547, 447 (1992).

[8] C. Fuchs, H. Lenske, and H. H. Wolter, Phys. Rev. C 52, 3043 (1995).

[9] S. Kubis and M. Kutschera, Phys. lett. B 399, 191 (1997).

[10] H. Mueller and B. D. Serot, Nucl. Phys. A606, 508 (1996).

[11] H. Muether, M. Prakash, and T. L. Ainsworth, Phys. Lett. B 199, 469 (1988).

[12] H. Huber, F. Weber and M. K. Weigel, Phys. Rev. C 50, R1287 (1994).

[13] L. Engvik, M. Hjorth-Jensen, E. Osnes, G. Bao, and E. Ostgaard, Phys. Rev. Lett. 73, 2650 (1994).

[14] N. K. Glendenning, Phys. Rev. Lett. 57, 1120 (1986); Z. Phys. A 326, 57 (1987); 327, 295 (1987).
[15] I. Bednarek and R. Manka, Int. J. Mod. Phys. D. 10, 607 (2001).

[16] F. Hofmann, C. M. Keil, and H. Lenske, Phys. Rev. C 64, 025804 (2002).

[17] D. Kaplan and A. Nelson, Phys. Lett. B 175, 57 (1986).

[18] J. C. Collins and M. J. Perry, Phys. Rev. Lett 34, 1353 (1975).

[19] I. N. Mishustin, M. Hanauske, A. Bhattacharyya, L. M. Satarov, H. Stoecker, and W. Greiner, Phys. Rev. Lett. B 552, 1 (2003).

[20] V. A. Ambartsumyan and G. S. Saakyan, Soviet. Astron. 4, 187 (1960).

[21] A. A. Tyaokin, Sov. J. Nucl. Phys. 22, 89 (1976).

[22] N. K. Glendenning, Astrophys. J. 293, 470 (1985).

[23] F. Weber and M. K. Weigel, Nucl. Phys. A493, 549 (1989); A505, 779 (1989).

[24] M. Rufa, J. Schaffner, J. Maruhn, H. Stocker, W. Greiner, and P. G. Reinhard, Phys. Rev. C 42, 2469 (1990).

[25] S. K. Ghosh, S. C. Phatak, and P. K. Saha, Z. Phys. A 352, 457 (1995).

[26] T. Bressani and F. Iazzi, Nuovo. Cim. A 102, 597 (1989).

[27] D. J. Millener, C. B. Dover, and A. Gal, Phys. Rev. C 38, 2700 (1988). 
[28] S. Bart et al., Phys. Rev. Lett. 83, 5238 (1999).

[29] E. Friedman, A. Gal, and C. J. Batty, Nucl. Phys. A579, 518 (1994).

[30] N. K. Glendenning, Phys. Rev. C 64, 025801 (2001).

[31] S. Balberg, I. Lichtenstadt, and G. B. Cook, Astro. J. Suppl. Series 121, 515 (1999).

[32] P. Khaustov et al., Phys. Rev. C 61, 054603 (2000).

[33] I. N. Filikhin and A. Gal, Phys. rev. C 65, 041001(R) (2002).

[34] H. Takahashi et al., Phys. Rev. Lett. 87, 212502 (2001).

[35] J. K. Bunta and S. Gmuca, Phys. Rev. C 68, 054318 (2003).

[36] H.Q. Song, R.K. Su, D.H. Lu, and W.L. Qian, Phys. Rev. C 68, 055201 (2003).

[37] J. Schaffner, C. B. Dover, A. Gal, C. Greiner, and H. Stoecker, Phys. Rev. Lett. 71, 1328 (1993); Ann. of Phys. (N.Y.) 235, 35 (1994).

[38] J. Mares, E. Friedman, A. Gal, and B. K. Jennings, Nucl. Phys. A594, 311 (1995).

[39] J. Schaffner-Bielich and A. Gal, Phys. Rev. C 62, 034311 (2000).

[40] G. Q. Li, R. Machleidt, and R. Brockmann, Phys. Rev. C 45, 2782 (1992).

[41] C. H. Lee, T. T. S. Kuo, G. Q. Li, and G. E. Brown,
Phys. Rev. C 57, 3488 (1998); Phys. Lett. B 412, 235 (1997).

[42] H. Huber, F. Weber, and M. K. Weigel, Phys. Lett. B 317, 485 (1993); Phys. Rev. C 51, 1790 (1995).

[43] H. Huber, F. Weber, M. K. Weigel, and Ch. Schaab, Int. J. of Mod. Phys. E 7, 301 (1998).

[44] I. Vidana, A. Polls, A. Ramos, L. Engvik, and M. HjorthJensen, Phys. Rev. C 62, 035801 (2000).

[45] J. Schaffner and I. N. Mishustin, Phys. Rev. C 53, 1416 (1996).

[46] G. Mao, P. Papazoglou, P. Hofmann, S. Schramm, H. Stoecker, and W. Greiner, Phys. Rev. C 59, 3381 (1999).

[47] T. Waas, M. Rho, and W. Weise, Nucl. Phys. A617, 449 (1997).

[48] G. Q. Li, C. H. Lee, and G. E. Brown, Nucl. Phys. A625, 372 (1997); Phys. Rev. Lett. 79, 5214 (1997).

[49] R. Barth et al., Phys. Rev. Lett. 78, 4007 (1997).

[50] The original motivation of strange meson introduction was the simulation of strong YY interaction. In spite of the recent experimental proof of a weak $\Lambda \Lambda$ interaction in ${ }_{\Lambda \Lambda}^{6} \mathrm{He}$ 34] there still remain reasons for their incorporation [36]. 
TABLE III: Dependence of the binding energy per nucleon $E_{b}(\mathrm{MeV})$ and the nuclear pressure $p\left(\mathrm{MeV}^{\mathrm{fm}}{ }^{-3}\right)$ on the baryon density $\rho_{B}\left(\mathrm{fm}^{-3}\right)$ in $\beta$-equilibrated matter for all of the parameterizations considered in this article. The onset of hyperons causes a significant change especially in the nuclear pressure behavior.

\begin{tabular}{|c|c|c|c|c|c|c|c|c|c|c|c|c|c|c|c|c|}
\hline \multirow[b]{2}{*}{$\rho_{B}$} & \multicolumn{2}{|c|}{ MA } & \multicolumn{2}{|c|}{ MB } & \multicolumn{2}{|c|}{$\overline{\mathrm{LA}}$} & \multicolumn{2}{|c|}{$\overline{\mathrm{LB}}$} & \multicolumn{2}{|c|}{$\overline{\mathrm{HA}}$} & \multicolumn{2}{|c|}{$\overline{\mathrm{HB}}$} & \multicolumn{2}{|c|}{$\overline{\mathrm{HC}}$} & \multicolumn{2}{|c|}{$\mathrm{HD}$} \\
\hline & $E_{b}$ & $p$ & $E_{b}$ & $p$ & $E_{b}$ & $p$ & $E_{b}$ & $p$ & $E_{b}$ & $p$ & $E_{b}$ & $p$ & $E_{b}$ & $p$ & $E_{b}$ & $p$ \\
\hline 0.02 & 6.28 & 0.06 & 6.45 & 0.06 & 3.87 & 0.03 & 3.15 & 0.02 & 3.03 & 0.02 & 2.85 & 0.01 & 3.14 & 0.02 & 2.93 & 0.02 \\
\hline 0.04 & 8.46 & & & & & & & & & & & & & & & \\
\hline 06 & 8.64 & & & & & & & & & & .29 & & & & 54 & .20 \\
\hline 08 & 8.94 & & & & 49 & & & & 56 & & 57 & & & & & 49 \\
\hline 0.1 & 9.46 & & & & & 0.78 & 36 & & 17 & & 7.31 & & 8.31 & 0.81 & 7.59 & 0.98 \\
\hline 0.12 & 10.39 & & 37 & & 10.62 & & & & & & & & & & & \\
\hline 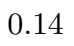 & 1.81 & & .89 & & & 2.02 & & & & 1.86 & 12.18 & .82 & & & 2.28 & .66 \\
\hline 16 & 3.65 & & 2.9 & 2.87 & & 2.96 & & & & & 15.25 & & & & 5.15 & .88 \\
\hline 18 & .87 & 3.88 & 32 & 4.21 & 17 & 4.16 & & & 76 & 3.49 & 8.68 & .86 & & 3.65 & 8.32 & 5.38 \\
\hline 0.2 & 42 & & & & & & & & & & & & & & & .23 \\
\hline 22 & 1.26 & & & & & & & & & & & & & & & 47 \\
\hline 24 & 4.34 & & & & & 9.82 & & & & & .12 & & & & 61 & .17 \\
\hline 26 & 27.65 & 11.49 & 27.79 & 11.94 & 29.66 & 12.48 & 92 & & & & 35.99 & & & & & \\
\hline קค & 1.13 & (2) & & & 49 & - - & & & & & 41.26 & & & & & \\
\hline & 1.79 & & & & & & & & & & & & & & & \\
\hline 32 & 8.58 & & & & & 9 & & & & & & & & & 27 & \\
\hline 34 & 42.49 & 22.98 & 98 & 21 & & 36 & & & & & 57 & & & & & \\
\hline 36 & 6.52 & 26.42 & 47.03 & & 51.41 & 32.17 & & & & & & & & & & \\
\hline 38 & 0.64 & & & & & & & & & & & & & & 72.54 & \\
\hline 0.4 & 4.84 & & & & & 6 & & & & & 59 & & & & 90.09 & \\
\hline 42 & 59.12 & 9 & 61 & & 99 & 19 & & & & & & & & & 16 & .86 \\
\hline .44 & 63.45 & 42.24 & & & & 142.38 & 94.76 & & & 23 & 15 & & & & 88 & 1 \\
\hline 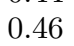 & & & & & & & & & & & & & & & & \\
\hline .48 & 76.46 & & & & & & & & & & & & .79 & & & \\
\hline 0.5 & 87.46 & 35 & 76 & & .86 & 89 & 14 & & 18 & 49 & .54 & 25 & .77 & .53 & .30 & 1.08 \\
\hline 0.52 & 98.72 & 2.09 & 8.83 & 1.26 & 2.28 & 192.86 & 7.35 & 202.69 & 38 & 245.77 & 234.58 & 308.91 & 158.61 & 253.38 & 210.36 & 285.31 \\
\hline 0.54 & 109.90 & & & & & & & & & & & & & & & \\
\hline 0.56 & 0.88 & & .96 & & & & & & & & & & & & 252.29 & \\
\hline 0.58 & 1.58 & 7.62 & .67 & 177.76 & 3.14 & 218.05 & 0.33 & 22 & & & 299.96 & & 67 & .69 & 272.99 & 346.33 \\
\hline 0.6 & 2.00 & 4.59 & 2.09 & 184.71 & 5.94 & 224.73 & 13.79 & 238.28 & 233.15 & & 321.51 & & 231.46 & & 293.48 & 367.04 \\
\hline 0.62 & 2.12 & & .21 & & & 23 & & & & & & & & & .79 & \\
\hline 0.64 & 161.93 & 197.69 & 162.01 & .51 & 220.38 & 236.64 & 239.46 & 254.68 & 267.15 & 344.56 & 368.37 & 431.83 & 266.17 & 349.28 & 333.92 & 410.4 \\
\hline
\end{tabular}

